\title{
La utilización del podcast como herramienta pedagógica. Caso de estudio de la asignatura Teoría y Ecología de los Medios Audiovisuales.
}

\section{Raúl Terol Bolinches ${ }^{\mathrm{a}}$, Nadia Alonso López ${ }^{\mathrm{b}}$ y Miguel Pareja Aparicio}

${ }^{a}$ Departamento Comunicación Audiovisual, Documentación e Historia del Arte, Universitat Politècnica de València rautebo@upv.es; 'bepartamento Comunicación Audiovisual, Documentación e Historia del Arte, Universitat Politècnica de València naallo1@har.upv.es; y Departamento de Ingeniería de Sistemas y Automática, Universitat Politècnica de València mipaap@isa.upv.es.

\begin{abstract}
The methodology of the flipped classroom changes the way of understanding teaching, taking better advantage of the times we share in the classroom and distributing more optimally the work outside of it. On the other hand, in recent months we have seen a remarkable growth in audio consumption on the Internet. The podcast, as an example of this, is conceived as audio on demand with the main intention of ending the fugacity of the sound message, making it possible for the listener to go to the audio resource as many times as he wishes.

In the present work we have considered the possibility of using the podcast as a pedagogical tool, so that its use in eminently theoretical subjects can be of great help to students. Thus, focusing on the subject Audiovisual Theory and Ecology, second year of the Degree in Audiovisual Communication of the $U P V$, we start with a quantitative analysis to know how the implementation of this tool can fit into the development of the subject.
\end{abstract}

Keywords: Podcast, Learning, on demand, Audio online, Flipped classroom, Really Simple Syndication

\section{Resumen}

La metodología de la clase inversa, oflipped classroom, cambia la forma de entender la docencia, aprovechando mucho mejor los tiempos que compartimos en el aula y distribuyendo de manera óptima el trabajo fuera de ella. Por otra parte, en los últimos meses hemos observado un crecimiento notable en consumo de audio en Internet. El podcast, como ejemplo de ello, se concibe como audio a la carta con la principal intención de acabar con la fugacidad del mensaje sonoro, haciendo posible que el oyente pueda acudir al recurso auditivo las veces que desee.

En el presente trabajo nos hemos planteado la posibilidad de utilizar el podcast como herramienta pedagógica, de tal manera que su empleo en asignaturas eminentemente teóricas pueda servir de gran ayuda a los alumnos. Así pues, centrándonos en la asignatura Teoría y Ecología de los Medios 
La utilización del podcast como herramienta pedagógica. Caso de estudio de la asignatura Teoría y ecología de los Medios Audiovisuales.

\begin{abstract}
Audiovisuales, de segundo curso del Grado en Comunicación Audiovisual de la UPV, se parte de un análisis cuantitativo para conocer cómo puede encajar la implementación de esta herramienta en el desarrollo de la asignatura.
\end{abstract}

Palabras clave: Podcast, Enseñanza, A la carta, Audio online, Docencia inversa, Sindicación de contenidos

\title{
Introducción
}

Podcast fue considerada palabra del año en 2005 para el Oxford University Press, solo un año después que apareciese. Su nombre parece tener relación con el dispositivo móvil creado por Apple, el iPod, y el término anglosajón broadcasting, emisión. Con el paso de los años, su perfeccionamiento ligado a la hiperconectividad que presentan las nuevas generaciones de estudiantes, ha hecho que hablemos de un crecimiento notable del consumo de audio online. Nuestro país, según el estudio de la agencia IAB, se sitúa en primer lugar por lo que respecta a la escucha de contenidos sonoros en Internet.

Esto nos ha hecho replantearnos la posibilidad de entender el podcast como una herramienta pedagógica que sirva al docente como manera de conectar con su público, es decir, sus alumnos. Los últimos años han crecido el número de herramientas web para fomentar el aprendizaje desde la misma, especialmente vídeos en los que el profesor enumera una serie de contenidos que aborda en la asignatura. En algunos casos, la imagen no aporta demasiada información ni amplía en exceso aquello que se está escuchando con la voz del profesor. Por ello, en el siguiente trabajo planteamos una serie de cuestiones técnicas y teóricas para abordar la presencia del podcast en el proceso de enseñanza-aprendizaje.

Además de proporcionar unas bases para comprender qué es el podcasting y cómo implementarlo en el aula, hemos considerado oportuno conocer cuál es la impresión que los alumnos pueden tener de esta innovación. Por este motivo, se ha realizado una encuesta a los que se encuentran matriculados en la asignatura Teoría y Ecología de los Medios Audiovisuales del Grado de Comunicación Audiovisual en el Campus de Gandia de la Universitat Politècnica de València. Planteando, además, la elaboración de varios podcasts en los que se abordaran contenidos que se encuentran en la guía docente de la asignatura.

El hecho de que el alumno utilice el podcast en las asignaturas en las que está matriculado puede reforzar el estudio del contenido eminentemente teórico, pudiendo ser abordado en el caso de la metodología Flipped Classroom, o docencia inversa, antes de la sesión presencial que el profesor ofrecerá en la universidad. Así como el alumno también podrá acudir al recurso sonoro tantas veces como lo desee y sea necesario, pudiendo escuchar el contenido mientras sale a correr o realiza actividades cotidianas.

El objetivo de nuestra investigación es implementar el podcast como herramienta de estudio por parte del alumnado en la asignatura Teoría y Ecología de los Medios, visto lo comentado anteriormente y el auge del audio en Internet, combinado con la implantación de la docencia inversa en algunas de las asignaturas en la Universitat Politècnica de València. Además,

(c)) EY-NC-ND 2019, Universitat Politècnica de València 
como se trata del Grado en Comunicación Audiovisual, otro de los objetivos es proponer a los alumnos que implementen los conocimientos adquiridos en las asignaturas de Expresión y Locución Oral, Proceso de Realización Radiofónica y Grabación y Edición de Sonido, y sean capaces de producir sus propios podcasts que el resto de los compañeros escuchará. Para conocer los efectos de la utilización del podcast como herramienta de innovación docente realizaremos una encuesta a nuestros alumnos con la que mediremos el grado de satisfacción del alumno ante dicha implantación.

\section{El origen del Podcast}

En los albores del presente siglo es cuando aparece el Podcast por primera vez, fruto de las distintas evoluciones generadas por las Tecnologías de la Información y las Comunicaciones (TIC). Adam Curry, periodista de la MTV y bloguero, conversaba con Dave Winner, informático y desarrollador del software Really Simple Syndication (RSS), para lanzarle la propuesta de que su sistema de sindicación de contenidos no se circunscribiese únicamente al texto, sino que ofreciese la posibilidad de indexar desde ese mismo fichero RSS cualquier tipo de archivo multimedia. Fue por ello, que Winner generó la posibilidad de añadir ficheros de audio a un RSS mediante la etiqueta enclosure, que le permitió incluir una canción a una de las entradas de su blog (Gallego, 2010: 75).

Durante los primeros años, con el auge de su utilización por parte de los blogueros, aparece una corriente que aboga por el perfeccionamiento de esta tecnología y por la posibilidad de que el usuario pueda disponer de los recursos sonoros de una manera automatizada, transfiriéndose directamente al reproductor MP3. La técnica del podcasting se había convertido en una realidad, con el paso del tiempo y la evolución de esta herramienta informática, se convertiría en una herramienta que ganaría muchos adeptos y que en nuestros días presenta un crecimiento cada vez mayor en cuanto a usuarios generadores de podcasts como de los consumidores de este formato sonoro (Sellas, 2009: 157).

\subsection{Definición del Podcast}

Existen numerosos intentos desde la academia de definir el podcast, a continuación enumeraremos aquellas definiciones que hemos considerado más relevantes:

Por una parte, María Blanco señala que se trata de "un archivo digital, normalmente en formato MP3, que cualquier persona puede crear para su posterior colocación en la web, quedando así accesible para todo el público". A esto le añade que "es un formato que permite la sindicación de archivos de sonido, con un sistema RSS, que admite suscripción y descarga de forma automática y periódica". A la hora de comprender el podcast, cobra mucha importancia el término sindicación, que se refiere a que "no es necesario visitar otra página web individualmente para escuchar el mensaje, ya que la descarga se realiza directamente" (Blanco, 2012).

Por otra parte, Juan Ignacio Gallego destaca que el podcasting, "a nivel tecnológico, es la unión de dos tecnologías ya conocidas anteriormente como son el MP3 y el RSS. Lo que ha 
conseguido el RSS es permitir la suscripción a una fuente que automatiza la descarga de los archivos MP3”. Incide en la importancia de la sindicación de contenidos como la otra autora, destacando el MP3 como un tipo de fichero más estandarizado y de mayor uso entre la población, aunque reconoce que "el podcasting se puede realizar con cualquier tipo de archivo de audio y que esto no cambia en absoluto su realidad” (Gallego, 2010: 33-34)

Como podemos apreciar en las definiciones anteriores, Blanco y Gallego hablan de Podcast y de Podcasting y resulta interesante diferenciar ambos aspectos. En primer lugar, Podcasting sería la acción de distribuir aquellos contenidos sonoros por Internet mediante la tecnología de la sindicación de contenidos, para lo que va a ser fundamental la incorporación de un fichero de audio en un feed. Esto es, según Toni Sellas, la dirección de un documento en Internet que ha sido generado mediante lenguaje XML, es por ello por lo que los podcasts se consideran feeds con contenido sonoro, que se encuentra incluido en la etiqueta enclosure de la que hablábamos (Sellas, 2009: 181).

Pero no solo nos encontramos ante un medio que converge y que aúna audio, Internet y dispositivos móviles, sino que tenemos ante nosotros una tecnología que rompe el esquema clásico de la comunicación radiofónica y que ha obligado a la radio a cambiar y a reconsiderar su punto de vista sobre las audiencias, los modos de consumo, la producción y la distribución, un completo cambio en la manera de hacer las cosas (Berry, 2006: 144). El mensaje, emitido a través de la radio, era efímero. La fugacidad del mensaje siempre ha sido una de las características que han acompañado a la radio desde su concepción, pero con el surgimiento del podcast se pone fin a esta percepción. Podemos acudir tantas veces como queramos para escuchar un programa y hacerlo desde cualquier lugar.

\subsection{Aparición del término Podcast}

El primero en utilizar el término podcast, propiamente dicho, fue Ben Hammersley. Lo hizo un 12 de febrero de 2004, en un artículo que tituló "Audible revolution" en el diario The Guardian. En su texto analizaba algunos de los casos existentes, por aquel momento, de distribución de contenidos sonoros, con cierta periodicidad, a través de los blogs. Uno de los casos fue el de Christopher Lydon, quien distribuía entrevistas que giraban en torno a la política. El ejemplo de la empresa Audible, quienes dejaron la venta de audiolibros para dedicarse a la distribución de programas de radio bajo demanda también captó la atención de Hammersley en su artículo. Proyectos como estos estaban gestando un fenómeno que todavía no había sido bautizado.

El periodista británico apunta como posibles los términos de audioblogging y podcasting, cuyas características principales residían en una interactividad más rápida y efectiva entre emisor y receptor y en la capacidad de reproducir los programas descargables donde, cuando y como quiera.

Existen diversas teorías sobre los orígenes del término podcast, de entre las que destacan las siguientes acepciones (Gallego, 2010: 77): 
- Por un lado, se señala que vendría derivado de la suma de iPod con Broadcasting. Apple no se encuentra ligada al origen de esta tecnología, pero sí en su evolución. El desarrollo del podcast en su plataforma iTunes supondrá un punto de inflexión en la evolución del podcast.

- Pod, cápsula en inglés, sumado con Broadcasting. Esto haría referencia a las cápsulas sonoras que se distribuyen en Internet, los podcasts.

- Personal o Publico on demand, junto con broadcasting, donde se recogería la principal característica del podcast de ser un audio bajo demanda.

- Personal Option Digital, sumado al concepto anglosajón de emisión que se repite en los otros casos. Esta fue la interpretación realizada por Doc Searls, editor del Linux Journal. Con esta redefinición del término podcast ponía especial atención a las posibilidades de elección personal que ofrece en contraposición de la emisión convencional.

La llegada del podcast a nuestro país se produce de la mano del periodista valenciano José Antonio Gelado, quien a finales de 2004 publicaría su primer podcast titulado "Comunicando podcast", en donde analizaba temas estrechamente vinculados con la comunicación, Internet y las entonces nuevas tecnologías (Gallego, 2010: 146; Sellas, 2009:166). Para el propio Gelado, el podcasting supone un gran cambio en la distribución de los contenidos audiovisuales y en la manera en la que la audiencia accede a los mismos.

\section{Desarrollo de la innovación: Aplicación del Podcast en la docencia}

La generación Z, todos aquellos nacidos a partir del año 2000, se caracteriza por ser una generación alfabetizada digitalmente, que presenta una gran facilidad para dominar la tecnología, pero que también manifiesta una gran dependencia de esta. Son ciudadanos de aquel mundo digital que Nicholas Negroponte auguraba en 1995. Nuestros alumnos actuales pertenecen a esta generación, por lo que los podcasts van a facilitar en gran medida el proceso de enseñanza-aprendizaje, aunque su dominio de la tecnología no va a implicar que se encuentren familiarizados y asimilen de una manera óptima los contenidos de nuestro temario en este formato sonoro (Piñeiro, 2012: 4).

\subsection{Flipped classrom, o la docencia inversa}

La denominada clase inversa (flipped classroom) es un método pedagógico que pretende modificar los roles del profesor y del alumno, tanto dentro como fuera del aula. Sustituye las clases magistrales en las aulas por prácticas, trabajos en equipo u otras dinámicas educativas $\mathrm{y}$ traslada fuera del aula los contenidos para que el alumno los pueda seguir a su propio ritmo.

De esta forma, los contenidos los aprende el alumno mediante el uso de materiales multimedia como vídeos o ficheros de audio, que se adecuen a los contenidos del temario a desarrollar, pudiendo resolver dudas o utilizar dichos contenidos en las horas de clase presenciales, aumentando así la participación del alumno en clase.

El objetivo es que las actividades de aprendizaje sencillas sean realizadas por los alumnos fuera del aula (como observar, memorizar, resumir, etc.), y en el aula dedicarlas a la 
realización de actividades más complejas (como razonar, examinar, priorizar, argumentar, proponer, etc.), las cuales requieren de la ayuda y experiencia del docente, pudiendo pasar de un aprendizaje pasivo de la metodología tradicional a un aprendizaje más activo.

Por una parte, en el aula el docente actúa de guía o facilitador del proceso de aprendizaje y atendiendo a la diversidad, además de realizar una retroalimentación a los equipos o grupos de alumnos, o incluso individualmente. Finalmente, establece espacios para la coevaluación y autoevaluación. Mientras, en el aula, el alumno realiza actividades (debates, exposiciones orales, informes, diarios de aprendizaje, cuaderno de trabajo, etc.), de las cuales recibe una retroalimentación por parte del docente y compañeros/as.

Por otra parte, fuera del aula el docente debe diseñar y planificar el proceso de aprendizaje integrando distintas estrategias o modelos de enseñanza (aprendizaje basado en proyectos, aprendizaje basado en retos, aprendizaje cooperativo y colaborativo, etc.). Todo ello requerirá del diseño de las actividades a realizar fuera del aula (ver vídeos, audios, documentos, enlaces, cuestionarios, etc.) y selección de las tecnologías para obtener ese aprendizaje activo, incluyendo actividades de evaluación que faciliten el aprendizaje del alumno. Mientras, fuera del aula, el alumno debe acceder a las actividades o recursos facilitados por el docente, y realizar las actividades propuestas. Con ello adquirirá los conocimientos que tendrá que aplicar en las actividades a realizar en el aula.

Se puede apreciar que esta metodología requiere de un mayor trabajo previo por parte de los docentes para definir tanto las actividades a realizar fuera y en el aula por parte del alumno, así como facilitar y/o elaborar los materiales multimedia, para la consulta por parte de los alumnos, los cuales deberán realizar un trabajo previo a la asistencia a las clases para un mejor aprovechamiento de las horas en el aula.

Esta metodología pretende servir de apoyo para conseguir las competencias transversales de la UPV establecidas en la asignatura de Teoría y Ecología de los Medios Audiovisuales:

- Innovación, creatividad y emprendimiento: innovar para responder satisfactoriamente y de forma original a las necesidades y demandas personales, organizativas y sociales con una actitud emprendedora.

- Responsabilidad ética, medioambiental y profesional: actual con responsabilidad ética, medioambiental y profesional ante uno mismo y los demás.

Puesto que los contenidos conceptuales los podrá seguir el alumno mediante el uso de los recursos disponibles, las clases presenciales se dedican a debatir, razonar y examinar los contenidos que el alumno ha de asimilar para superar la asignatura y la realización de actividades como debates, presentaciones orales, análisis de textos audiovisuales $u$ otras propuestas en esta línea en la que se emplearán diversos recursos audiovisuales.

\subsection{Recursos tecnológicos para la docencia}

Para poder implementar la metodología de la docencia inversa, está relacionada directamente con las tecnologías de la información y la comunicación (TIC), que consiste en los diversos medios o recursos tecnológicos a los cuales, tendrán acceso los alumnos para poder seguir los contenidos que tienen que revisar previamente a las clases presenciales. En el caso 
concreto de la asignatura de Teoría y Ecología de los Medios Audiovisuales se pretende que se dispongan de varios podcasts, archivos digitales de audio, que el alumno puede descargar de internet, escuchándolos en el momento y lugar que lo desee, tanto en su propio ordenador como en otro dispositivo portátil.

En consecuencia, el disponer de los archivos de audio en todo momento, permite al alumno el acceso a la información en cualquier sitio y momento, modificando de esta manera el contexto de enseñanza y educación, convirtiendo así cualquier dispositivo fijo (ordenador, etc.) o móvil (teléfono, etc.) en una herramienta de aprendizaje, puesto que el único requisito será tener acceso a internet o disponer de un elemento de almacenamiento externo, en donde previamente se haya descargado el fichero de audio.

Aunque los podcasts pueden ser de varios formatos, el formato elegido es el mp3, por ser el más extendido y es posible reproducirlo desde cualquier dispositivo portátil. Además, se requiere de un software que permita su creación, y posteriormente exportación a un archivo mp3, de entre las alternativas, el elegido es Audacity (https://audacity.es/) que se trata de un software libre y gratuito para distintas plataformas: Windows, MAC o Linux; permitiendo grabar utilizando un micrófono conectada a la entrada de línea de la tarjeta de sonido disponible en cualquier equipo informático, realizando la digitalización y posterior procesado, para finalmente exportar a otro formato de audio, en este caso mp3.

Audacity, se trata de una aplicación sencilla de manejar con una interfaz muy intuitiva, que incluye las herramientas imprescindibles para editar ficheros de audio de calidad.

Algunos de los aspectos a seguir a la hora de elaborar un podcast con los contenidos teóricos, son los que se indican a continuación, bajo la premisa que el alumno no se desconcentre y pierda la naturaleza del mensaje:

- Elaborar/disponer de un guion: sistematizar los contenidos, enfatizando sobre las principales ideas a recalcar.

- Elección del micrófono: se debe disponer de un audio nítido para que se escuche correctamente y se permita captar la atención del alumno, para ello es de vital importancia el elemento captador, en este caso el micrófono.

- Distribución en unidades o temas de audio: al dividir el contenido será más fácil de controlar la información disponible, y, además, permite al alumno planificar su propio aprendizaje.

- Procesado o complementos del podcast: se puede añadir música o sonidos, para mantener la atención del alumno, siendo cauteloso para no recargar el contenido pudiendo llegar a distraer o confundir a los alumnos.

- Utilizar una voz acorde al contenido: utilizar una voz entusiasta y motivante para que pueda transmitir emociones, para que el alumno pueda seguir el mensaje, realizando énfasis en las ideas que se desee resaltar, evitando voces monótonas o muy lineales que pueden llegar a aburrir. 
- El tiempo de duración es recomendable que sea de 4 a 10 minutos, evitando audios muy largos, puesto que pueden provocar la pérdida del interés por parte de los alumnos.

El alumnado tiene que tener acceso a los ficheros de audio, por lo tanto, se requerirá de una plataforma digital que permita colgar contenidos, para que puedan ser descargados o consultados vía streaming.

En el caso de la Universidad Politécnica de Valencia (UPV) se dispone de la plataforma de teleformación PoliformaT, que ayuda al docente a tutorizar una asignatura, en concreto se destaca la herramienta Recursos, que consiste en un almacén para alojar información para el alumno pueda consultar en cualquier momento: apuntes, artículos, lecturas, vídeos multimedia, etc., y contenidos, que consiste en un apartado destinado a la confección de material para manejarse como si se tratará de una web.

\section{Resultados. Caso de estudio: Asignatura Teoría y Ecología de los Medios Audiovisuales}

La asignatura a la que aplicaremos el podcast como herramienta para el aprendizaje es Teoría y Ecología de los Medios Audiovisuales, asignatura semestral que se imparte en segundo curso del Grado en Comunicación Audiovisual. Se trata de una asignatura eminentemente teórica, como su propio nombre indica, en la que se estudia fundamentalmente la relación de los medios audiovisuales con el entorno social. Para ello, en las sesiones presenciales se exponen los conceptos teóricos de manera que se genera un debate entre los alumnos y con el propio profesor acerca de los mismos. Asimismo, durante las clases se realiza el análisis de textos audiovisuales relacionados con los conceptos estudiados y presentaciones orales de parte de la materia a cargo de los propios estudiantes, preparadas previamente por ellos mismos con apoyo de bibliografía y en las que pueden emplear los recursos audiovisuales que consideren.

Es a raíz de estas presentaciones orales cuando pensamos que se podrían emplear estos contenidos preparados por los propios alumnos como recurso para el estudio para facilitar el aprendizaje. Después de estudiar diversas opciones, surge la idea de elaborar un podcast como complemento a apuntes, bibliografía y demás recursos para el estudio. Esto se debe, como se ha señalado en líneas anteriores, al crecimiento que ha experimentado el consumo de audio a través de Internet $^{1}$, a las posibilidades que ofrece esta tecnología para su reproducción, ya que solo requiere un dispositivo de almacenamiento externo y una conexión a Internet, y al hecho de que, al ser archivos de audio, pueden escucharse en cualquier momento y lugar sin ser necesaria la atención a la imagen, por lo que puede compaginarse con otra actividad como conducir o hacer deporte. A todo ello, añadimos el trabajo de las

\footnotetext{
${ }^{1}$ Según el Estudio de Audio Online elaborado por la Asociación de la publicidad, el marketing y la comunicación digital en España (IAB), el 44\% de los españoles escucha audio online, lo que sitúa a España a la cabeza de Europa, por delante de países como Francia, Italia, Reino Unido y Alemania.
} 
competencias transversales de la asignatura y el empleo de los conocimientos adquiridos en otras asignaturas como Proceso de Realización Radiofónica, Grabación y Edición de Sonido o Expresión y Locución Oral, que se imparten en el primer curso del Grado en Comunicación Audiovisual. De manera gradual, en los siguientes cursos, implementaremos la utilización de esta innovación docente con el fin de mejorar la calidad en el proceso de enseñanzaaprendizaje. Para el presente curso hemos partido de la implantación de dos podcasts a modo experimental.

\subsection{Encuesta y resultados}

Como primer paso para implementar este recurso para el aprendizaje y la docencia, queremos conocer la predisposición de los alumnos de Teoría y Ecología de los Medios Audiovisuales respecto al podcast y su empleo como herramienta para el estudio. Para tal fin, elaboramos una encuesta empleando la herramienta Google Forms con los ítems que podemos observar en la tabla 1.

Tabla 1. Ítems de la encuesta sobre la utilización del podcast como herramienta pedagógica

\begin{tabular}{|c|c|}
\hline Preguntas & Opciones de respuesta \\
\hline \multirow[t]{3}{*}{ ¿Eres usuario de podcast? } & Sí \\
\hline & No \\
\hline & A veces, pero no con mucha frecuencia \\
\hline \multirow{2}{*}{$\begin{array}{l}\text { ¿Piensas que puede ser útil utilizar el podcast } \\
\text { como recurso para el aprendizaje? }\end{array}$} & Sí \\
\hline & No \\
\hline \multicolumn{2}{|l|}{ Argumenta brevemente tu respuesta anterior } \\
\hline \multirow{2}{*}{$\begin{array}{l}\text { ¿Utilizarías este recurso para estudiar los } \\
\text { contenidos de esta asignatura? }\end{array}$} & Sí \\
\hline & No \\
\hline \multicolumn{2}{|l|}{ Argumenta brevemente tu respuesta anterior } \\
\hline \multirow[t]{2}{*}{$\begin{array}{l}\text { ¿Crees que el podcast lo debería hacer el } \\
\text { profesor o los alumnos guiados por el } \\
\text { profesor? }\end{array}$} & $\begin{array}{l}\text { Puede ser un trabajo interesante para el alumno } \\
\text { como actividad transversal porque ponemos en } \\
\text { práctica } \\
\text { conocimientos de otras asignaturas. }\end{array}$ \\
\hline & $\begin{array}{l}\text { Es el profesor quien ha de elaborarlo } \\
\text { como recurso docente }\end{array}$ \\
\hline
\end{tabular}


Como vemos, en primer lugar, nos interesa conocer si los alumnos son usuarios de podcast para conocer el grado de penetración de esta tecnología entre ellos. Después, si consideran este recurso de utilidad para su estudio y si lo emplearían en el caso de disponer de él. Por último, queremos aproximarnos a la conveniencia de que sean los mismos alumnos quienes preparen el podcast guiados por el profesor, o el mismo profesor como el resto de los recursos elaborados para la asignatura.

\subsubsection{Resultados de la encuesta realizada al alumnado}

Obtenemos 67 respuestas de un total de 75 alumnos matriculados, lo cual significa que el $89,3 \%$ del alumnado responde al formulario. Por tanto, podemos considerar los resultados como representativos de los alumnos de la asignatura.

Respecto a los resultados obtenidos, encontramos que solo un 16,4 por ciento de los alumnos son usuarios habituales de podcast, siendo superior el porcentaje de alumnos que no lo son con un 26,9 por ciento. Sin embargo, la mayoría, un 56,7 por ciento, lo utilizan, pero con poca frecuencia. Así, podemos concluir que la mayor parte de los alumnos están familiarizados con esta tecnología. Lo vemos en la figura 1.

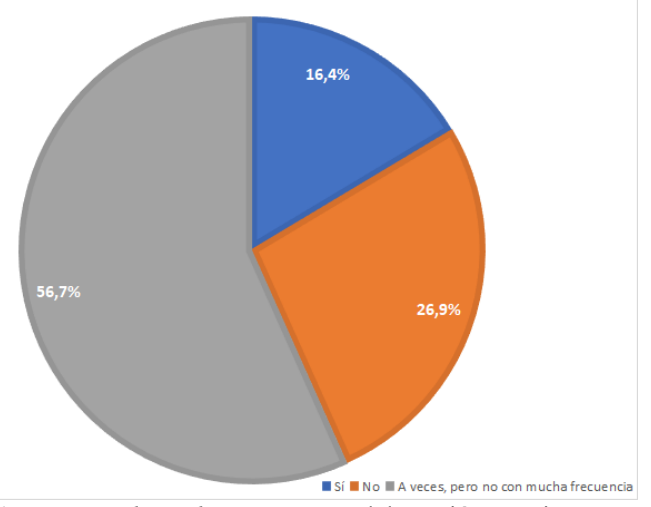

Fig. 1 Usuarios de Podcast. Fuente: Elaboración propia

Nos fijamos ahora en los resultados de la segunda pregunta planteada y comprobamos como la amplia mayoría de los alumnos consideran que la utilización del podcast sería adecuada como recurso para el aprendizaje. Observamos en el gráfico 2 como el 91 por ciento de los alumnos responde afirmativamente. 


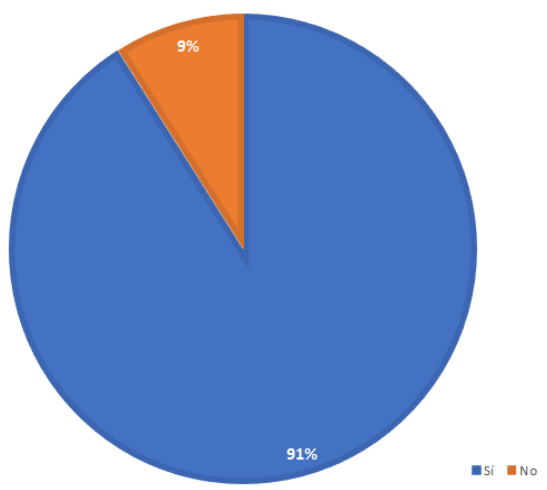

Fig. 2 Utilidad del podcast como recurso para el aprendizaje. Fuente: Elaboración propia

Respecto a los argumentos a favor, la mayor parte de comentarios se refieren a la posibilidad de poder reforzar los contenidos de una forma sencilla y amena, además con un material elaborado por los mismos alumnos que les permite poner en práctica también lo aprendido en otras asignaturas. Los alumnos que contestan de forma negativa muestran su preferencia por otros recursos, como los videoapuntes.

En cuanto a la pregunta sobre si los alumnos utilizarían el podcast como herramienta para el estudio, más del 86 por ciento sí lo harían, como se puede ver en el gráfico 3.

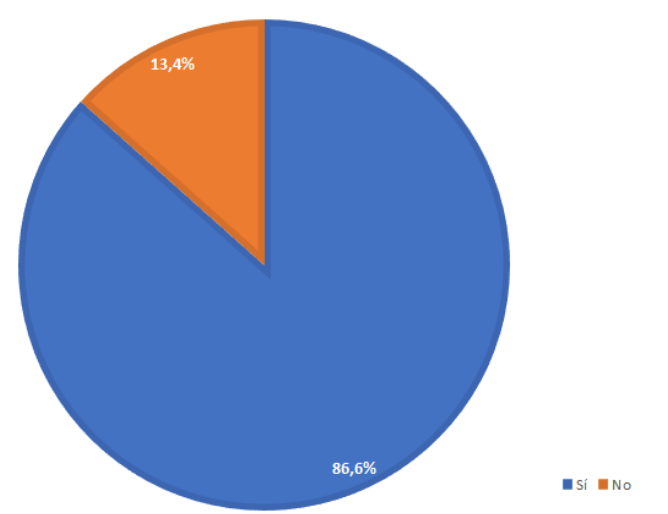

Fig. 3 Los alumnos utilizarían el podcast como herramienta de estudio. Fuente: Elaboración propia

Los argumentos a favor se sitúan principalmente en la línea de que escuchar los contenidos les puede ayudar a asimilar y retener mejor los conceptos. Por el contrario, los alumnos que contestan negativamente afirman que les desconcentrarían y prefieren estudiar leyendo y debatir y comentar los conceptos en clase.

En este punto, consideramos importante destacar algunos datos. Es el caso de los 16 alumnos que responden que no son usuarios de podcast, pero sí lo consideran útil como herramienta para el estudio. De ellos, solo tres no lo utilizarían para estudiar a pesar de estar a favor de su 
implementación como recurso. Por otra parte, 4 de los alumnos usuarios de podcast pero con poca frecuencia no lo emplearían para estudiar, pero dos de ellos lo consideran un recurso útil para el aprendizaje. Destacamos también que solo dos alumnos han contestado negativamente a todas las cuestiones, es decir, no les parece un recurso útil y no lo emplearían para el estudio.

Por último, al referirnos a la elaboración del podcast, los alumnos consideran que deberían ser ellos mismos guiados por el profesor quienes elaboren este recurso, como podemos observar en el gráfico 4.

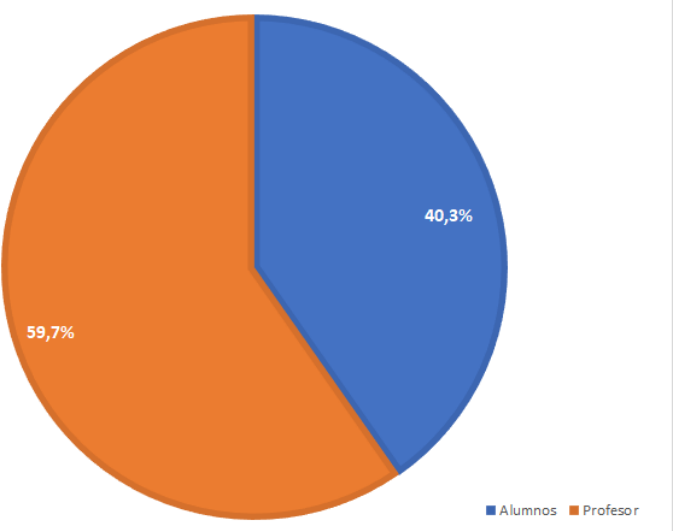

Fig. 4 Recurso elaborado por el profesor o por los alumnos guiados por el profesor. Fuente: Elaboración propia

De esta manera, podemos concluir al calor de los datos que la amplia mayoría de los estudiantes consideran de manera positiva la introducción del podcast como recurso para la docencia y el aprendizaje que han de elaborar ellos mismos guiados por el profesor. Estos resultados nos permiten pasar a la siguiente fase y llevar a la práctica la creación de podcast con contenidos de la asignatura.

\subsection{Planificación de actividades}

Para la elaboración del podcast, comenzamos realizando una selección de los contenidos y estableciendo una duración de este. A partir de las exposiciones realizadas en clase, planteamos dos podcasts, cada uno de ellos elaborados por los alumnos que realizaron dichas exposiciones. Decidimos que serán sobre:

- El concepto de Hegemonía según Antonio Gramsci y ponerlo en relación con los medios.

- Cool Media y Hot Media, según la distinción realizada por Marshall McLuhan.

Fijamos la duración de los podcasts en 4 minutos cada uno de ellos. 
A continuación, planificamos las siguientes actividades:

- Elaboración del contenido y su adaptación a la estructura de un guion de podcast. Para ello, se proporciona a los alumnos bibliografía y otros recursos, como podcast de ejemplo.

- Entrega para revisión y corrección. La profesora responsable de la asignatura de Teoría y Ecología de los Medios Audiovisuales y el profesor responsable de la asignatura Proceso de Realización Radiofónica revisan y corrigen el trabajo de los alumnos antes de la grabación. Una vez revisado, el trabajo se devuelve a los alumnos.

- Planificación y ensayo de la grabación de los podcasts. Se establece un día y un horario para la grabación de los podcasts en el estudio de radio del Campus, que se reserva previamente por el profesor. Los alumnos preparan y ensayan la grabación en una tutoría previa con los profesores.

- Grabación de los podcasts. La realizan los mismos estudiantes que han elaborado el podcast en el estudio de radio del Campus con la supervisión de los profesores responsables de Teoría y Ecología de los Medios y de Proceso de Realización Radiofónica. Los compañeros que quieran pueden asistir hasta completar el aforo del estudio (25 personas).

- Edición y posproducción de los podcasts. A cargo de los estudiantes bajo la supervisión de los profesores responsables de Teoría y Ecología de los Medios y de Proceso de Realización Radiofónica.

- La profesora responsable de Teoría y Ecología de los Medios Audiovisuales los publica en la plataforma Poliformat correspondiente a la asignatura para que todos los alumnos tengan acceso a estos.

\section{Conclusiones}

Nos encontramos en un momento en que la tecnología forma parte de nuestra cotidianidad sin que muchas veces reparemos en ello. En este sentido, el consumo de contenidos audiovisuales online y bajo demanda y los recursos tecnológicos que ello implica puede ser una excelente herramienta para la docencia y el aprendizaje. Por ello, consideramos que la implementación del podcast en asignaturas teóricas es muy positiva, tanto para alumnos como para docentes.

Los dos podcasts diseñados como prueba experimental en este curso han tenido un número medio de escuchas de 189 el referente a Antonio Gramsci y 126 el que giraba en torno a los conceptos vinculados a Marshall McLuhan. Para su difusión entre los alumnos se subió el fichero MP3 resultante de la grabación del podcast a la página web archive.org, con la intención de difundir el feed entre los alumnos de tal manera que pudiesen descargarlo en su móvil más fácilmente. 
Al preparar estos contenidos, los alumnos investigan sobre los conceptos vistos en clase a partir de bibliografía, apuntes y otros recursos, con lo que, consecuentemente, aprenden y asientan los conocimientos en la materia. Esta investigación, a su vez, tiene una finalidad práctica y relacionada con su formación transversal en Comunicación Audiovisual, al elaborar un recurso como el podcast en el que también ponen en juego conocimientos en otras áreas como la radio y lo que ello implica a nivel técnico, guion o locución, entre otros. Asimismo, este contenido tiene un gran valor para los estudiantes, ya que lo han elaborado ellos mismos y, a su vez, es una herramienta con la que reforzar los contenidos de la asignatura a través del audio. En este punto, nos referimos a los resultados del formulario mencionados anteriormente, en el que el 91 por ciento de los alumnos consideran el podcast útil para el aprendizaje, y un 86,6 por ciento lo utilizarían para el estudio.

Por otra parte, la implementación de este recurso acerca el podcast a alumnos que no son usuarios de este. De hecho, un 20 por ciento de los alumnos declaran en el formulario que no usan el podcast, pero sí lo consideran una herramienta útil para el aprendizaje. De estos, un 80 por ciento afirma que lo utilizaría para el estudio.

En el caso concreto de la asignatura Teoría y Ecología de los Medios Audiovisuales, se ha implementado esta herramienta de forma experimental en el curso 2018-19. A lo largo de los siguientes cursos evaluaremos el rendimiento de los alumnos a medida que vayamos incorporando mayor número de podcast al repositorio y valoraremos si los estudiantes obtienen mejores notas con la implementación de esta herramienta de innovación docente. Por este motivo, no se contempla en la guía docente de la asignatura un porcentaje para la evaluación de este trabajo de los alumnos, pero sí se abre la posibilidad de tenerlo cuenta en próximos cursos dado que ha sido una experiencia positiva y con buen resultado tanto para alumnos como para profesores.

\section{Referencias}

Alba Fernández, Jesús; Cabanilles, Constantino; Vidal, Anna y del Rey, Romina. (2017). Aplicando Flipped Teaching en Física del Grado en Ingeniería de Sistemas de Telecomunicación, Sonido e Imagen. 10.4995/INRED2017.2017.6864.

ASOCIACIÓN DE LA PUBLICIDAD, EL MARKETING Y LA COMUNICACIÓN DIGITAL EN ESPAÑA (IAB). (2018). Estudio de Audio Online. Recuperado de: <https://iabspain.es/> [Consulta: 15 de marzo de 2019].

Blanco, M. (2012). Análisis del conocimiento de podcasts en España e Hispanoamérica. Caracterización de los usuarios y factores que determinan su consumo como nuevo medio de comunicación (Inédita). Tesis doctoral defendida en la Universidad Pontificia de Salamanca Recuperado de $<$ https://www.tdx.cat/handle/10803/147260> [Consulta: 11 de marzo de 2019]

Gallego Pérez, J. I. (2010). Podcasting: Nuevos modelos de distribución para los contenidos sonoros. Barcelona: UOC.

López Collazo, Zeidy; Rodríguez-Jiménez, Andrés y Dávila Valdés, Yosdey. (2018). Experiencia pedagógica en la implementación de la clase invertida en el proceso de formación académica. 10.13140/RG.2.2.12977.68961.

(c)) BY-NC-ND 2019, Universitat Politècnica de València 
Piñeiro-Otero, T. (2012). Los podcasts en la educación superior. Hacia un paradigma de formación intersticial. Revista Iberoamericana De Educación,58(1), 1-12. Recuperado de $<$ https://rieoei.org/RIE/article/view/1462> [Consulta: 10 de marzo de 2019]

Sellas, T. (2009). La voz de la Web 2.0. Análisis del contexto, retos y oportunidades del podcasting en el marco de la comunicación sonora (Inédita). Tesis doctoral defendida en la Universidad Internacional de Catalunya, Palafrugell. Recuperado de $<$ http://www.tdx.cat/handle/10803/9351> [Consulta: 10 de marzo de 2019]

Serrano Pastor, R.M. y Casanova-López, Oscar. (2018). Recursos tecnológicos y educativos destinados al enfoque pedagógico Flipped Learning. REDU. Revista de Docencia Universitaria. 16. 155. 10.4995/redu.2018.8921.

Solano Fernández, Isabel $\mathrm{M}^{\mathrm{a}}$ y Sanchez Vera, $\mathrm{M}^{\mathrm{a}}$ Mar (2010). Aprendiendo en cualquier lugar: el podcast educativo. Píxel-bit, Revista de medios y educación. No36 Enero 2010 pp. 125-139.

Terán Delgado, Laura y González Hernández, Amador Jesús (2014). Una propuesta didáctica: el uso del podcast como objeto de aprendizaje en la educación superior. Revista iberoamericana para la investigación y el desarrollo educativo (RIDE). ISSN 2007-7467.

Vidal Meló, A.; Boigues Planes, FJ.; Estruch Fuster, VD. (2017). Prácticas de Matemáticas 2: de la clase tradicional a la clase inversa. En In-Red 2017. III Congreso Nacional de innovación educativa $\mathrm{y}$ de docencia en red. Editorial Universitat Politècnica de València. 502-516. doi:10.4995/INRED2017.2017.6841 